\title{
Robert Gloton: un militant pédagogique français entre progressisme et conservatisme
}

\author{
Noëlle Monin
}

Résumé:

Entre 1945 et 1957 l'inspecteur primaire Robert Gloton, militant du GFEN, enrôle une grande partie des instituteurs de sa circonscription dans des innovations pédagogiques fédérées par le Groupe Coopératif d'Education Nouvelle (GCEN) qu'il fonde et préside. Son parti pris pour un enseignement qui se voulait en rupture avec la forme scolaire académique de l'époque et fondé sur une critique sans complaisance de l'encyclopédisme, tient-il du progressisme comme il le prétend luimême? Le bimestriel Échanges, qu'il crée alors qu'il est en poste dans le département de l'Eure (1948-1957) rend compte de la démarche innovatrice des maîtres. L'analyse de contenu des 44 numéros de cette revue nous amène à conclure à une certaine préservation de positions conservatrices combinée à une posture innovante.

Mots clés:

innovations pédagogiques; progressisme; conservatisme; encyclopédisme. 


\title{
Robert Gloton: a French pedagogical activist between liberalism and conservatism
}

\begin{abstract}
Between 1945 and 1957 primary inspector Robert Gloton, a GFEN activist, enrolls a large part of the teachers in his constituency in pedagogical innovations federated by the Cooperative Group of New Education (GCEN), which he founded and presides over. Was his bias for an education that wanted to break with the academic form of the time and based on an uncompromising criticism of encyclopaedism as progressive? The bimonthly Echange has his initiative and published while he is stationed in the department of Eure (1948-1957) reports the innovative approach of the masters. The content analysis of the 44 issues leads us to conclude that there is a certain preservation of the conservative positions of teachers possibly combined with an innovative posture.
\end{abstract}

Key words: educational innovations; progressivism; conservatism; encyclopedism.

\section{Robert Gloton: un activista pedagógico francés entre el progresismo y el conservadurismo}

Resumen: Entre 1945 y 1957, el inspector primario Robert Gloton, activista de GFEN, inscribe a una gran parte de los maestros en su circunscripción en innovaciones pedagógicas federadas por el Grupo Cooperativo de Nueva Educación (GCEN), que fundó y preside. ¿Se inclinó por una educación que quería romper con la forma académica académica de la época y se basó en una crítica inflexible del enciclopedismo como progresista? El intercambio bimestral tiene su iniciativa y se publica mientras está estacionado en el departamento de Eure (1948-1957) informa sobre el enfoque innovador de los maestros. El análisis de contenido de los 44 temas nos lleva a concluir que existe una cierta preservación de las posiciones conservadoras de los maestros, posiblemente combinada con una postura innovadora.

Palabras clave: innovaciones educativas; progresismo; conservadurismo; enciclopedismo.

\section{Robert Gloton: um ativista pedagógico francês entre o progressismo e o conservadorismo}

Resumo: Entre 1945 e 1957 o Inspetor primário Robert Gloton, militante da GFEN, agregou uma grande parte dos professores da sua circunscrição em inovações pedagógicas federadas pelo Grupo Cooperativo de Educação Nova (GCEN) que ele funda e preside. A sua aposta por um ensino que pretenda romper com a forma escolar académica da época baseia-se numa crítica sem complacência ao enciclopedismo, será que se pode considerar progressista como ele próprio o pretendia?O bimestral Echanges, que ele cria enquanto ele está como inspector no departamento do Eure (1948-1957) mostra a abordagem inovadora dos professores. A análise de conteúdo dos 44 números desta revista leva-nos a concluir que há uma certa preservação de posições conservadoras, combinada com uma postura inovadora.

Palavras-chave: inovações educativas; progressismo; conservadorismo; enciclopedismo. 
Dès son premier poste d'inspecteur primaire en 1945 et jusqu'aux expériences du $20^{\text {ème }}$ arrondissement de Paris en 1962, Robert Gloton a eu la possibilité de mettre en place dans sa circonscription une autre conception de l'école. Son ambition visait "une autre pédagogie pour une autre école [afin que le maître] renonce à son pouvoir dominateur...[car] il ne peut "décoloniser» l'enfant s'il n'est pas "désaliéné»" (Gloton, 1979, 179). II s'agit de comprendre dans quelle mesure cette initiative s'affronte à des idées adverses, s'inscrit dans le registre d'une alternative, d'une réflexion enseignante contre-hégémonique (Robert, 2014), qui peut s'entendre sous le terme progressisme. Sur quels terreaux et réseaux Gloton a-t-il pu s'appuyer pour pouvoir développer une manière de penser l'école autrement, et expérimenter celle-ci hors des voies classiques? En quoi la pensée, qu'il affiche comme progressiste, peut-elle trouver une expression dans l'école de son époque et s'inscrire dans une visée désaliénante telle qu'il aime à le dire, a fortiori quand la forme scolaire reste celle de l'école qu'il critique et quand sa fonction d'inspecteur le lie à une certaine orthodoxie? Cette pensée pourrait-elle relever paradoxalement du conservatisme que Gloton dénonce verbalementpar ailleurs? Ce questionnement sera ici mis à l'épreuve à travers l'analyse de la revue Échanges, créée par Gloton, journal de bord des instituteurs innovateurs de sa circonscription de l'Eure entre 1950 et 1957.

\section{Entre un itinéraire banal et un devenir d'exception}

Entré à l'École Normale d'Auteuil en 1921, Gloton devient instituteur en 1924, puis inspecteur de circonscription en 1945 jusqu'à sa retraite en 1971. En 1962, quand les écoles du $20^{\text {ème }}$ arrondissement de Paris deviennent expérimentales à son initiative, le système scolaire français n'est pas particulièrement réputé pour son avant-gardisme. Tel était déjà le cas dans les circonscriptions où il a mis en place ses premières innovations entre 1945 et 1957. II faut donc se demander comment un inspecteur de circonscription a pu réussir à obtenir l'aval de l'administration pour organiser des écoles d'un genre nouveau, ou du moins avoir les coudées franches pour procéder à "une révolution copernicienne" dans ces écoles, comme il l'écrit.

\section{Un héritage fécond}

Né en 1906 dans le quartier populaire de Ménilmontant, de sa famille il dit: "la mécanique de précision [côté maternel] c'est l'aristocratie, le polissage [côté paternel [c'est la roture" (Gloton, 1979, p. 16). Bien que Gloton insiste sur sa condition modeste, il va bénéficier d'un milieu et d'un climat familial particulièrement stimulants. Son père avait le certificat d'études, sa mère le brevet et était maîtresse de couture dans les écoles de la ville de Paris. Excellent élève du primaire, sa famille le pousse à devenir instituteur, en particulier sa mère, en contact privilégié avec le monde scolaire 
dans son travail. Reçu premier du canton au certificat d'études à 12 ans, il entre en 1918 à l'Ecole primaire supérieure Arago à Paris. En 1921, il est admis à l'Ecole Normale d'Auteuil d'où il sortira instituteur en 1924.

Malgré la rigidité de l'Ecole Normale (EN) qu'il dénonce, il sait trouver des voies singulières. De son passage normalien, il ne retient que les côtés négatifs: "on ignorait superbement l'enfant"; "il s'agissait de fortifier la puissance du pouvoir sur l'élève et une méfiance doctrinale à son égard"; "nous étions l'objet d'une surveillance constante, tatillonne, inquisitoriale" (Gloton, 1979, pp.50-53). Cependant l'obligation d'être interne constitue un temps de capitalisation culturelle par la formation reçue, mais également par les rencontres peu banales qu'il sait faire. Dans sa promotion, il se lie d'amitié avec certaines personnalités hors du commun, comme André Jolivet qui deviendra une notoriété du monde de la musique: "la musique nous rapprochait" (Gloton, 1979, p.55). Ses amitiés se nouent également avec le fils d'un couple d'instituteurs, dont il admire le bon rang d'entrée et de sortie de l'EN.

Pendant ce temps normalien, on peut penser que Robert Gloton a été sensibilisé au mouvement de l'éducation nouvelle. La Ligue internationale pour l'éducation nouvelle fondée à Calais trois ans plus tôt (1921) a une influence certaine sur les milieux pédagogiques. En cette même année, on trouve des informations sur d'autres manières d'enseigner dans l'hebdomadaire Le journal des instituteurs où les élèves maîtres peuvent lire:"Formons des hommes, des hommes à la mémoire moins encombrée et à l'intelligence plus ouverte, des hommes capables de se gouverner sans le secours d'autrui, et non des encyclopédies vivantes que le fatras de leurs connaissances empêche de voir le monde réel. N'ayons plus le fétichisme de la «mémorisation des leçons»". Ces propos sont appuyés par des références à Rousseau. On y trouve également un éloge de la classe promenade, la suggestion que le jardin du maitre soit une annexe pédagogique de l'école. Une rubrique bibliographique porte à la connaissance des instituteurs des publications relatives à des expériences pédagogiques d'un type nouveau. L'école libertaire lasnaïa Pollana fondée par Tolstoï est présentée comme ayant des résultats "excellents". De la même manière, les principes essentiels de la pédagogie Montessori sont exposés favorablement. Un numéro de 1922 se réfère à l'ouvrage de Decroly sur la méthode des centres d'intérêt. "C'est un admirable livre", ces propos de Ferdinand Buisson concluent la présentation. D'autre part, à l'Ecole Normale, comment ne pas imaginer que Gloton ait été sensibilisé aux revendications de la jeunesse communiste, particulièrement véhémente dans cet établissement à partir du milieu des années 1920 (Thomas, 1998)? A la même époque, les techniques Freinet ne sont pas ignorées des milieux pédagogiques. Freinet lui-même écrit dans L'école émancipée, vecteur du syndicalisme révolutionnaire, très actif à l'École Normale d'Auteuil. En même temps, les écoles nouvelles sous la houlette de Desmolins en France, de Reddie en Angleterre ou 
de Geheeb en Allemagne, bien que critiquées, sont célèbres pour enseigner autrement, Il est donc probable que ces mouvements aient été une ressource pour sa pensée même s'il n'en dit pas un mot dans son autobiographie.

\section{L'instituteur étudiant à la Sorbonne en 1934}

Devenu instituteur en 1927, Gloton était condamné à une carrière dans le premier degré. Rien ne laisse supposer dans ses écrits qu'il ait été retenu pour préparer l'école supérieure de Saint Cloud afin de devenir lui-même professeur d'École Normale, ainsi qu'il était proposé aux plus brillants des normaliens d'Auteuil. Entreprendre des études supérieures pour un instituteur de l'époque restait exceptionnel, encore fallait-il bénéficier de conditions, elles aussi exceptionnelles, avoir un poste dans une ville universitaire et réussir à suivre les cours en n'ayant qu'un jour de liberté. En poste à Paris, Gloton a pu suivre les cours de la Sorbonne dès 1934, son aspiration à accéder à l'enseignement secondaire étant forte: "[..]des diplômes de l'enseignement supérieur pouvaient me faire accéder à un poste de cours complémentaire et pénétrer ainsi, même par la petite porte, dans l'enseignement secondaire" (1979, p. 90). A la Sorbonne, il aura comme professeurs Bouglé, Bayet, Fauconnet. En 1937, il suit les cours du psychologue Henri Wallon qui vient d'être nommé au Collège de France: "ses cours me passionnèrent" écrit-il (Gloton, 1979, p. 96). Celui-ci devient son maître à penser. Gloton se présente finalement au concours de l'inspection primaire en 1939. Ajourné à l'oral, il garde le bénéfice de l'admissibilité.

\section{Dans un oflag d'officiers, il lit Albert Thierry}

Prisonnier dans les camps nazis, il va savoir tirer le meilleur parti de ces "5 ans dans un tunnel" (1979, p. 112). Je trouvais de quoi préparer mon oral d'inspection avec des professeurs et une documentation que je n'aurais sans doute pas eus à Paris". Bénéficiant du traitement des officiers, il va côtoyer des prisonniers de milieux intellectuels auprès desquels il enrichir sa cultura. C'est à la bibliothèque du camp que Gloton a accès à "des recherches très complètes sur l'éducation nouvelle" (p. 122). II découvre en particulier le texte d'Albert Thierry Réflexions sur l'éducation (Thierry, 1923). "J'avais sauvé de la captivité le texte d'Albert Thierry, le bon instituteur de Vosves ...extrait de sa troisième lettre de Vosves d'un intérêt unique et qui convenait admirablement à mon projet" (Gloton, 1979, p. 143). Le rôle majeur que donne Gloton à l'ouvrage de Thierry dans la construction de sa pensée occulte l'inspiration commune avec Freinet. Même si Thierry et Freinet ne partagent pas le même point de vue pédagogique, leur ambition voisine vise à mettre en place une école pour les enfants du peuple fidèle à la culture populaire en vue d'une transformation de la société. 


\section{Le militant du GFEN}

Enfin dans cet itinéraire qui forge sa pensée pour une autre école, il faut noter le rôle spécifique qu'a joué pour Gloton son entrée au GFEN (Groupe français d'Education nouvelle) en 1950. La promotion des orientations du mouvement qu'il a su faire dans ses premiers postes d'inspecteur lui valurent la reconnaissance de Wallon qui préside alors le GFEN "Henri Wallon me demanda de partager la vice-présidence avec Fabre" (p. 188). A partir de ce moment, il fait partie des délégations du GFEN conduite à deux reprises par Wallon en URSS où il rencontrera, entre autres, le psychologue russe Léontiev. II se rendra en Autriche en 1951 pour des rencontres sur l'éducation. Ces voyages seront d'un double profit. D'abord, Gloton s'inspire du modèle scolaire soviétique dont il dit que "ces ouvertures sur le monde extérieur apportaient une nourriture substantielle à mon travail d'inspecteur" (p. 193). Ensuite, il lui revient un rôle de missionnaire de ce modèle grâce aux multiples conférences de propagande militante qu'il donnera à son retour: "[...] ce que j'y appris, je dus le rapporter dans de nombreuses conférences qui me furent demandées à mon retour en Normandie et à Paris" (p. 189).II se défend vigoureusement néanmoins d'un quelconque engagement au Parti communiste, tout en spécifiant que les autorités du GFEN de l'époque étaient des communistes engagés avec lesquels il était en rapport permanent. Wallon était directeur de la revue La Pensée, les inspecteurs de la Seine, Fabre vice-président du GFEN, et Madame Seclet-Riou étaient au comité de parrainage de la revue. "Il était communiste dans la tête mais ne pratiquait pas", précise un ancien instituteur du GCEN (extrait d'entretien, 2018).

C'est dans cet itinéraire, dans sa capacité à tirer le meilleur parti des situations qu'il rencontre, dans les réseaux communistes qu'il a fréquentés et dans une puissante aspiration à se distinguer dans le corps des instituteurs que Gloton a puisé sa pensée critique.

\section{La pensée de Gloton construite sur la critique des classiques de l'école}

La pensée de Gloton est construite sur une dénonciation de l'école qu'il qualifie d'autoritaire telle qu'il dit l'avoir vécue en tant qu'élève. Dans un texte autobiographique, il cherche à convaincre le lecteur de la nocivité d'une telle pédagogie (Gloton, 1979). II dénonce l'autoritarisme du maître et l'exercice de sa domination. De sa scolarité primaire, il met essentiellement en évidence l'abus de pouvoir de ses maîtres: "Mon professeur de maths....Zizim nous terrorisait dès qu'il pénétrait dans la classe...Zizim dévissait le capuchon de son stylo, assurait ses lunettes pour consulter son registre... un long silence...le cœur qui battait la chamade...enfin le nom tombait" (Gloton, 1979, p. 40). Il en va de même quand il occupe ses premiers postes d'instituteur: "Le jour du certificat d'études, les maîtres chargés de sa préparation se réunissent comme examinateurs, c'est l'occasion de confidences, la plupart portant sur les difficultés de tirer 
quelque chose de ces gosses, sur leur paresse et leur mauvaise volonté...l'heure était venue, ils allaient payer le prix et déjà ils savouraient leur vengeance" (Gloton, 1979, p. 85). La soumission de l'élève à l'autorité, sa moindre considération par le maître font l'objet de sa part d'une critique récurrente. Tous les élèves dans ce cas étaient considérés comme des "paumés irrécupérables" (1979, p. 203). II voit dans le système de récompenses et de punitions une entrave aux véritables finalités éducatives de l'école: "un système où l'enfant est colonisé, un objet de dressage en vue d'objectifs à court terme, où l'institution existe pour elle-même avec les colis à son service sans objectif à long terme et [...]sans finalité éducative". La forme scolaire qui est en œuvre lui paraît définir un dressage des corps: il dénonce "[...] l'absurdité de garder les petits enfants assis sans bouger, sur les bancs trop hauts pour que leurs pieds touchent terre et qui doivent rester ainsi des heures les jambes pendant dans le vide" (Gloton, 1979, p.172); il estime que, des esprits "[...] on réclamait toujours par cœur des tables de multiplication et [que] l'on faisait toujours ces dictées inventées pour provoquer des fautes et témoigner de l'inégalité naturelle des hommes, les uns ne faisant jamais de fautes, d'autre en faisant à chaque mot" (p. 206). L'organisation de l'espace condamne selon lui le besoin de mouvement de l'élève:"[Dans] une cour de $22 \mathrm{~m} \times 24 \mathrm{~m}$, il n'est pas facile de faire tenir 200 élèves ensemble" (p. 83); " [...] Chacun [des maîtres] avait constamment devant les yeux sa propre classe, avec ses quatre murs, ces tables qui bloquaient l'activité et le besoin de mouvement" (Gloton, 1979, p. 150).

Pour Gloton, le système scolaire maintient également les maîtres dans un état "d'aliénation". Le processus prend de multiples formes: le modèle de la leçon type consistant à " préparer des leçons stéréotypées tirées des manuels pour les débiter en tranches" (p. 154); le modèle du bon maître pratiquant "I'interrogation de contrôle, l'exposé de la leçon nouvelle bien préparée, avec ses exercices d'application, la succession des devoirs à la maison et des leçons à apprendre" (p. 84); une forme scolaire immuable avec "[...]un cours magistral... où le professeur dans sa chaire, les élèves à leur table se regardent face à face dans une disposition matérielle qui concrétise le rapport de force, les inégalités fonctionnelles, l'impossibilité de la communication" (p. 38).

Dans son premier poste d'inspecteur rural dans la Marne en 1945, il dénonce de même l'autoritarisme de l'administration: "Tout ce qu'on pouvait faire pour le mauvais maître, c'était de lui infliger une sanction administrative, baisse de la note de mérite entraînant un retard à l'avancement, avertissement, blâme, déplacement d'office; son activité professionnelle devenait alors une entreprise itinérante de démolition scolaire" ( $p$. 138). Nommé inspecteur -professeur à l'Ecole Normale d'Auteuil en 1957, il constate: “j'allais dans les écoles d'application et je retrouvais le maître tout puissant” (p. 200). ॥ retient essentiellement la rigidité du système: "c'était d'abord apprendre à obéir et nos formateurs partageaient cette certitude que, pour nous plier à la règle d'obéissance, il fallait accentuer les marques du pouvoir et la méfiance à notre égard “ (p. 53). C'est 
ainsi qu'il résume la formation professionnelle de l'époque. Gloton est particulièrement incisif en caractérisant la profession d'instituteur de "prolétariat enseignant" (p. 86).

L'immobilisme et le conservatisme font partie des autres aspects scolaires qu'il déplore. Lorsqu'il parle des écoles de sa circonscription en 1947, on ne trouve rien de similaire avec l'attitude très révérencieuse d'Emilie Carles (1977) ou d'Edouard Bled (1977) vis-à-vis de l'institution scolaire. Ses propos sont plutôt accablants pour l'institution: "une maison pauvre et triste où rien n' accroche l'attention, pas mêmes les enfants... Partout la même vie ralentie au souffle court, où les jours passent à remplir les petites cases de l'emploi du temps réparti en tranches cloisonnées... École anonyme impersonnelle à l'image du bureau de poste ou de la petite gare de campagne" (Gloton, 1979, p. 176). C'est un système qu'il a l'intention de renverser en particulier en devenant inspecteur :" [...] une circonscription d'inspection primaire, par la liberté d'action qu'elle donne à son responsable, devait faire un foyer expérimental " (1979, p. 132); il insiste sur son intention transformatrice en évoquant son examinateur au jury d'oral d'inspection:” le cher homme ne savait pas qu'il était en train d'introduire le loup dans la bergerie ". Autrement dit, la sémantique de la dénonciation du conservatisme de l'institution scolaire, la volonté de faire table rase des principes qui maintiennent, selon Gloton, les instituteurs dans une position dominée, ne sont pas sans rappeler les orientations marxisantes du GFEN et le projet communiste pour changer l'école. Sans être un militant du parti, l'inspecteur a baigné dans cet univers au cours des années 1950.

\section{L'inspecteur missionnaire des pédagogies nouvelles}

Le choix de l'inspectorat a offert à Gloton une marge de liberté qu'il a su exploiter: “j'allais faire l'inspection....rechercher s'il était possible de faire pénétrer dans la forteresse bien close et puissamment structurée de l'instruction publique, l'esprit et les méthodes de cette éducation nouvelle dont je me sentais désormais [à son retour de l'oflag] l'adepte" (Gloton, 1979, p. 131). Ainsi dès son premier poste en 1945, dans la Marne il ambitionne de "[...] réaliser [...] «la révolution copernicienne de l'éducation» que voulait Claparède” (p. 140). Dans cette perspective, il exerce son inspectorat à la manière d'un missionnaire. II vise la transformation des mentalités des instituteurs de sa circonscription: "il fallait élaborer une stratégie d'approche des problèmes d'éducation pour qu'elle suscite petit à petit une transformation des mentalités" (1979, p. 142-143). Il se donne donc les moyens de cette stratégie en proposant, au moment des conférences pédagogiques, une rencontre aux instituteurs de sa circonscription. Cinquante d'entre eux répondent présents. Gloton leur remet la lettre d'Albert Thierry et procède à une explication de texte: "[...] ce fut un beau concert de protestation indignées...il était clair que mon texte explosif avait sur les maîtres un impact que n'aurait jamais produit la conférence pédagogique la plus persuasive" (1979, p. 145). La démarche revient à 
persuader les maîtres d'inventer collectivement une nouvelle manière de faire. Disciple de Wallon, dont il développe longuement les propos contenus dans De l'acte à la pensée, Gloton est convaincu par l'idée que "la pensée naît de l'action pour retourner à l'action" (p. 101), que l'homme a des ressources créatrices qui ne demandent qu'à s'exprimer. Bénéficiant de la sensibilisation obtenue par le texte de Thierry, il persuade une poignée d'instituteurs de constituer un groupe de recherche. L'objectif principal était de concevoir "I'élaboration d'un projet éducatif commun concernant l'adaptation de l'école à l'enfant et l'expérimentation concrète dans les écoles et les classes intéressées" (p. 148). Le cœur des méthodes nouvelles préconisées par Gloton consiste en des emprunts aux grands principes de l'éducation nouvelle et aux méthodes actives, glanés chez des auteurs de référence comme Rousseau, Claparède, Decroly, Montessori, Kerschensteiner, Wallon. II s'agit de promouvoir le tâtonnement expérimental, les centres d'intérêt, la vie coopérative, l'ouverture de l'école sur l'extérieur.

Quittant cette circonscription de la Marne pour en rejoindre une autre dans l'Eure en 1948, Robert Gloton profite à nouveau des conférences pédagogiques pour inviter les maîtres à une réflexion "Le résultat positif de ces premières conférences pédagogiques, ce furent nos rencontres libres de l'après-midi....j'y étais interpellé....sur mon monologue du matin et je pouvais voir comment mes coups bas avaient porté" (p.168). Fort de l'expérience de son poste précédent, il réussit rapidement à recréer un dispositif du même genre dont les retombées vont le couvrir de lauriers et l'installer dans une réputation d'inspecteur innovateur: "Robert Gloton est unique, presque insolite au sein de l'éducation nationale" (Vial, 1980). "Gloton, c'était un inspecteur extraordinaire." (extrait d'entretien, 2018) renchérit un ancien instituteur de sa circonscription. En 1950, le Groupe coopératif de pédagogie nouvelle des Andelys (GCEN) fédère des petites équipes cantonales d'instituteurs avec pour chacune un coordonnateur. II atteint 450 adhérents au milieu des années 1950, Gloton en est président jusqu'en 1957. L'objectif du GCEN est ambitieux puisqu'il est question de faire "table rase de la pédagogie orthodoxe" dénoncée comme aliénante pour l'enfant et le maître dans l'intention d'inventer une démarche innovatrice qui les libère. Pour Gloton, il s'agit d'organiser une "communauté en petites équipes" engagée "dans une production novatrice" (p.170). "Tout devait nécessairement tourner autour de la mise en œuvre des méthodes actives par lesquelles les enfants deviennent les artisans de leur propre formation... le passage du maître transmetteur ...à l'éducateur facilitateur des apprentissages...relation nouvelle de caractère démocratique avec reconnaissance de la personne de l'élève" (1979, p. 170). Le GCEN est organisé par un esprit coopératif fait d'échanges d'expériences entre les instituteurs du groupe ou avec ceux d'autres régions, voire d'autres pays. Le bi-mestriel Échanges, à l'initiative de Gloton, rend compte des activités du groupe dans les 44 numéros publiés entre 1950 et 1960. 


\section{La pensée de Robert Gloton entre progressisme et conservatisme}

Nous nous attacherons ici à saisir le débat entre conservatisme et progressisme porté par 39 numéros d'Échanges publiés pendant la période où Gloton est inspecteur dans la circonscription des Andelys-Vernon et président du GCEN, soit 1950-1957.

\section{Des orientations hors des classiques de l'école}

II s'agit pour Gloton de rallier les instituteurs de sa circonscription à une nouvelle manière de penser et de faire fonctionner l'école, en rupture avec l'habitus normalien et l'académisme scolaire. Gilles Laprévote en résume l'esprit qui "promeut le maître face aux livres, lui donne l'initiative puis le contrôle de l'activité de l'enfant" (Laprévote, 1986). Le mouvement nait au moment où Gloton entre au GFEN et multiplie les appels pour participer aux activités du mouvement parisien, auxquelles assistent certains membres du GCEN. Un long compte rendu des journées d'études du GFEN à Paris du 7 au 10 novembre 1953 est rédigé par la secrétaire du GCEN (n 18). La même année Gloton invite Favre, vice- président du GFEN, à en présenter les orientations aux journées pédagogiques de sa circonscription. L'année suivante Gal en sera le conférencier. En 1955, l'inspecteur d'académie de l'Eure assiste à l'assemblée générale du GCEN et en devient président d'honneur: "La marque d'estime qu'il vient de donner au GCEN est pour nous tous non seulement un honneur, mais un précieux encouragement"(n²9). En 1957, c'est au tour d'un Inspecteur Général de devenir le second vice-président. Une revue de lectures fait, dans la plupart des numéros publiés, le résumé d'ouvrages jugés importants. Celui de Maurice Debesse, Les étapes de l'éducation, est un exemple: "en 157 pages il [le livre de M. Debesse] donne une somme de renseignements sur la psychologie de l'enfant que tout éducateur se doit de connaître ( $\left.n^{\circ} 16, p .21-23\right)$. La rubrique bibliothèque liste les ouvrages recommandés comme ceux de Sartre, Merleau-Ponty, Camus, Rousseau, Cousinet, à côté de Rostand, Mérimée, La Fontaine, Vigny.

L'influence des théories walloniennes marque les finalités déclinées: "L'important c'est d'entreprendre" ( $\left.{ }^{\circ} 19, p .18\right)$ ou bien "L'enfant est en classe pour apprendre à penser" (n`2, p.33). Pour atteindre ce but, il faut développer l'esprit critique, ouvrir la voie de la recherche chez les élèves, rechercher leur équilibre psychique. Gloton dit "permettre aux maitres d'atteindre une connaissance intime de la vie psychique de ses élèves dans ce qu'elle a de plus inconscient et de plus déterminant" (n¹2, p.1-2) ou bien: "Le mot devient l'instrument de la pensée" ( $n^{\circ} 19$, p.17-19). L'enfant doit être aussi "pleinement heureux à l'école" (n¹2, p. 3). Le regard porté par les maîtres influencés par Gloton sur leurs élèves doit être celui du psychologue: "L'enfant possède en lui tout ce qui convient à sa propre construction" ( $\left.n^{\circ} 2, p .12\right)$ ou bien: "L'enfant ne se livre à une étude que lorsqu'il en a ressenti le besoin, c'est-à-dire lorsqu'il répond à une nécessité nouvelle de son adaptation", et encore: "La lecture viendra plus tard quand 
l'enfant en ressentira le besoin" (n`5, pp. 1-5). Changer le statut de l'enfant est donc un principe auquel s'attellent les membres du GCEN.

Les articles de Gloton lui-même dans la majorité des numéros sont d'incessants encouragements à l'expérimentation. Persuadé de l'inefficacité de la dictée, il a initié une expérience sur l'orthographe. Elle a été lancée dans 46 classes de la circonscription entre octobre 1952 et avril 1953. Dans 23 d'entre elles, les maîtres ont renoncé à cette épreuve traditionnelle. Les résultats sont exposés à grand renfort de pages de statistiques et de courbes. Gloton conclut: "la pratique de cet exercice à raison de deux heures par semaine ne donne pas de meilleurs résultats pour l'acquisition de l'orthographe que sa suppression et l'utilisation des deux heures en cause à des travaux d'expression écrite" ( $\left.n^{\circ} 17, p .1\right)$. Dans le même registre, un long exposé rend compte de l'expérience, Le Louvre itinérant initiée par le fils de Gloton, étudiant en histoire de l'art: "une exposition itinérante organisée par le centre international de l'enfance destinée à donner aux écoliers de Seine et Marne un aperçu des richesses du musée du Louvre. C'est pour nous l'occasion de nous interroger sur la portée de l'expérience. "Comment laisser aux enfants le souvenir le plus profond? L'acquisition de quelques reproductions servira mieux la classe que plusieurs visites de musées même à domicile. L'œuvre d'art doit être une réalité familière" (n¹8, p. 19-22).

Les enquêtes font également partie de la panoplie d'innovations mises en place au GCEN. Celle sur la sensibilité artistique de l'écolier a été conduite par Gloton dans 64 classes et a concerné 1024 élèves pendant l'année 1952-1953. Gloton préconise: "si nous voulons que leur sensibilité s'ouvre au monde merveilleux du beau, contribue à l'indispensable synthèse psychique de la personnalité, il faut donner à tous les enfants une éducation artistique dès leur plus jeune âge" (n¹8, p. 22). Le résultat de ces enquêtes constitue un appui pour inciter à rompre avec la forme traditionnelle de l'enseignement, ses finalités, ses contenus, ses objectifs.

\section{Une épistémologie pratique}

Le didacticien Sensévy (2011) considère que la conception de l'enseignement par les enseignants reviendrait à élaborer une sorte de théorie de la connaissance née de la pratique. C'est un point de vue qui peut s'appliquer à la conception de l'enseignement des instituteurs du GCEN. Nous donnerons quelques exemples de contenus au cœur de cette épistémologie pratique. Pour Gloton, "le texte libre correspond à la nouvelle orientation de l'enseignement de la rédaction ....il a sa place à tous les cours... le texte libre est une technique c'est-à-dire un instrument utilisé au développement de la langue écrite" (n`12, pp. 1-2). Le texte libre est aussi présenté comme un outil thérapeutique. Dans les articles rédigés sur ce sujet, les auteurs-instituteurs manipulent des concepts de la psychologie, voire de la psychiatrie dont la maîtrise s'avère sans doute partielle. Pour remplacer la dictée, Gloton propose la pratique du texte reconstitué: "Sans le secours du texte lu, l'enfant doit placer des étiquettes dans l'ordre pour reconstituer l'histoire. II 
ne peut y parvenir que s'il a lu avec intelligence et compris... Le procédé est auto-correctif c'est son premier avantage" (n¹1, pp. 13-19). Un instituteur ajoute: "exercice de réflexion, d'analyse, et de synthèse qui doit être sans aucun point commun avec un exercice mécanique de mémoire" ( $\left.n^{\circ} 13, p .3\right)$. Ce type d'exercice est complété par la lecture silencieuse: "Un fichier de lecture silencieuse individualisée est recommandable... Soit en séance de travail libre, soit lorsqu'ils disposent de quelques loisirs" (n¹1, pp.13-19).

Avec le texte libre, l'observation est une autre "clef de toutes les acquisitions" $\left(n^{\circ} 14,9\right)$ et devient une démarche majeure au GCEN. L'inspecteur novateur précise: "L'exercice d'observation est essentiel et seul procédé valable pour l'acquisition d'une connaissance, quelle qu'elle soit, à l'école primaire"( $n^{\circ} 14$, p. 3); "les procédés d'observation mis en œuvre conduisent à des inductions aussi aisées que celles qui résultent de l'observation des propriétés du rectangle....." ( $n^{\circ} 7$, p. 10-15). Les bénéfices se répercutent sur l'ensemble des apprentissages, ajoute une institutrice: "grâce à l'exercice d'observation l'enfant fait, sans effort, des progrès marqués en orthographe et arrive au second trimestre à rédiger seul de courts développements non seulement corrects mais parfois pleins de finesse et d'élégance" ( $\left.n^{\circ} 17,10\right)$.

La méthode globale fait aussi partie démarche qui se veut progressiste: "il ne peut être question de revenir en arrière et d'enseigner à lire le français comme les Égyptiens apprennent à déchiffrer les hiéroglyphes" ( ${ }^{\circ} 5$, p. 5-10) Les enquêtes des élèves s'ajoutent également aux principes fondamentaux censés être en rupture avec la " scolastique". II s'agit d'apprendre en liaison avec la réalité quotidienne, d'enraciner sa propre culture dans son environnement.

Du point de vue des supports retenus, l'imprimerie fait des adeptes parmi les maîtres du GCEN: "j'ai basé tout l'apprentissage du français sur l'imprimerie" (n6, p.3) ou les fichiers: "les livres de classe sont supprimés et remplacés par le fichier individuel qui est enrichi tout au long de la scolarité" (n5, pp.1-5). Gloton ajoute "ne jamais s'asservir au manuel, à son contenu et à son découpage. Le manuel n'est qu'un instrument de documentation à consulter" ( $n^{\circ} 14$, p.9). Bien qu'on ne trouve aucune référence ni allusion à Freinet dans Échanges, bien des aspects de la pédagogie de Gloton semblent converger avec ceux du fondateur de L'imprimerie à l'école. Un ancien membre du GCEN tient ainsi à préciser: "Gloton lui-même.... c'était pas... un fan de Freinet" (extrait d'entretien, 2018).

De toute évidence cependant, Gloton emprunte aux techniques Freinet sans en faire la même interprétation politique que le pédagogue de Vence. Ce n'est que 40 ans plus tard que Gloton, interrogé sur les origines du CGEN, acceptera de dire: "à l'époque c'était la transmission des connaissances. J'ai essayé de faire une organisation d'Education Nouvelle. J'ai fait 2 ans d'informations, de sensibilisation, on organisait des réunions, je leur expliquais Freinet" (Lethierry, 1986, p. 125). 


\section{Gloton et le conservatisme qu'il dénonce}

A lire attentivement l'ensemble des articles publiés dans la revue Échanges, les dimensions conservatrices inhérentes à la révolution pédagogique rêvée par Gloton ne peuvent cependant pas échapper au lecteur. Peut-on de ce fait s'autoriser à avancer l'idée d'une sorte de cécité de la inspecteur par rapport aux enjeux politiques de l'école traditionnelle? Cet aveuglement laisserait seulement libre cours à l'illusion de l'innovation pour changer l'école. C'est sans doute le seul rêve que son statut et sa position d'inspecteur pouvaient permettre sans risques encourus. Les quelques exemples qui vont suivre étaieront ce point de vue.

D'une manière générale les innovations pédagogiques dont les instituteurs du GCEN rendent compte se passent dans un cadre scolaire qui ne témoigne pas vraiment d'une réflexion enseignante contre-hégémonique comme Gloton le laissait entendre. Ainsi, en tant qu'inspecteur, il joue le rôle institutionnel imparti à son statut. II n'a pas abandonné les traditionnelles conférences pédagogiques, reprenant la démarche d'une leçon modèle qu'il expose longuement: "je m'étais proposé de présenter une leçon au CE, une au CM et une à la classe de FE. Voici les plans de ces leçons" ( $n^{\circ} 11, p$. 1). Dans un autre registre, les jugements disqualifiants portés par les maîtres sur leurs élèves des milieux modestes jalonnent certains articles, alors que l'influence de Gloton aurait dû les prémunir de s'exprimer ainsi "Fleuve nous a paru un mot de vocabulaire adapté seulement aux tout premiers élèves dans un milieu nettement plus cultivé que celui de la moyenne de la classe" ( $\left.n^{\circ} 13, p .15\right)$, "Combien .... de cas des débiles mentaux dénombre-t-on dans chacune de ces classes?" (n¹0, pp. 13-14). Ces jugements sont l'expression d'un habitus "institutoral" constant, il a été mis en évidence pour l'ensemble de la corporation par certains travaux de sociologie de l'éducation, à différentes époques (Bourdieu, Passeron 1970; Isambert-Jamati,1976; Testanière et Pinthiaux, 1979, Bourdieu, Champagne, 1992, Broccholichi, 1995, Merle,1996). Ces jugements n'épargnent pas non plus les familles: "une partie des indisciplinés, des paresseux se recrutent dans les familles désunies" ( $\left.n^{\circ} 19, p p .17-19\right) ;$ "je pense avec sympathie aux collègues exerçant dans certains centres industriels et dans certaines communes rurales déshéritées où sévissent l'alcoolisme, les taudis, et les maigres salaires d'ouvriers agricoles" ( $\left.n^{\circ} 10, p p .13-14\right)$.

II est intéressant de noter également une sorte d'hybridation des points de vue de ces maîtres innovateurs. A côté de leur tonalité anti-encyclopédiste forte, il n'est pas rare que certains instituteurs se fassent le chantre de méthodes traditionnelles condamnées par le GCEN: "II ne faut pas pour autant renoncer à meubler la mémoire. La faculté qu'ont les enfants d'ingurgiter est étonnante. Y renoncerons-nous sous prétexte que pour nous adultes, apprendre par cœur est à peu près impossible?" (n¹0, pp. 3-5); "Nous avons cru devoir nous appuyer sur un manuel -non pour en suivre aveuglément 
le texte- mais pour nous fier à sa progression" ( $n^{\circ} 13$, p. 14). La valorisation des meilleurs qu'ils célèbrent s'accorde avec leurs idées progressistes, a fortiori lorsque cette position favorable à l'excellence est proposée par Gloton lui-même:"La lecture individuelle est conduite par les meilleurs en lecture, ils liront facilement, ils donneront le ton, ils montreront l'exemple" ( $n^{\circ} 11$, p. 2). Malgré leur critique de l'autoritarisme, certains maîtres du GCEN peuvent aussi en exposer le bienfait. Si les hiérarchies sont critiquées dans ce groupe d'innovateurs partisans de plus d'horizontalité dans les rapports interpersonnels, Gloton lui-même préserve néanmoins son statut d'inspecteur, et maintient la hiérarchie verticale qu'il veut pourtant combattre. II est toujours désigné par les instituteurs du GCEN par la formule traditionnelle et déférente: "Monsieur l'inspecteur".

\section{Conclusion}

Le débat entre progressisme et conservatisme ouvert à propos des innovations pédagogiques mises en place dans les décennies 1950 et 1960 par Gloton pourrait nous amener à conclure à l'affirmation d'un esprit simplement réformiste, sans velléité subversive. L'analyse de contenu de la revue Échanges qui rend compte des pratiques préconisées par l'inspecteur Gloton met en évidence à la fois une préservation de certaines positions conservatrices, et la possibilité d'innover à l'intérieur de l'institution d'Etat, dans certaines limites cependant. Ainsi une forme de conservatisme et une forme de progressisme pédagogique sont-elles conduites à cohabiter. L'inspecteur a eu la possibilité de mettre en place des innovations pendant 30 ans de carrière, il a acquis une véritable célébrité dans le monde scolaire tant en France qu'à l'étranger. Missionnaire du mouvement progressiste GFEN, il en a distillé les orientations auprès d'instituteurs qu'il a su enrôler à sa cause. Son parti pris pour un enseignement en rupture partielle avec l'académisme institutionnel ne l'a pas amené néanmoins à se poser des questions sociologiques sur l'école et sur la fonction de sélection sociale qu'elle exerce, ni à se demander s'il suffit d'innover pour changer l'école.

\section{Références}

Arendt, H. (1972). La crise de la culture. Paris: Gallimard, Folio.

Bled, E. (1977). Mes écoles. Paris: le Livre de poche

Bourdieu, P. (1966). L'école conservatrice. Les inégalités devant l'école et devant la culture. Revue française de sociologie, VII, 325-347.

Bourdieu, P. \& Champagne, P. (1992). Les exclus de l'intérieur. Actes de la Recherche en Sciences Sociales, 91/92, 71-75.

Bourdieu, P. \& Passeron, J.C. (1970). La reproduction, éléments pour une théorie du système d'enseignement. Paris: Minuit. 
Broccolichi, S. (1995). Domination et disqualification en situation scolaire. In Cours Salies, P. La liberté pour apprendre. Paris: Syllepse.

Carles, E. (1977). Une soupe aux herbes sauvages. Paris: Le Livre de poche.

Gloton, R. (1979). Au pays des enfants masqués. Paris: Casterman.

Isambert-Jamati V. (1976). Les enseignants et la division sociale à l'école aujourd'hui. La pensée, 190, 17-32.

Laprévote, G. (1986). Les Ecoles Normales primaires en France:1879-1979; splendeurs et misères de la formation des maitres. Lyon: Presses universitaires de Lyon.

Merle, P. (1996). L'évaluation des élèves, enquête sur le jugement professoral. Paris: PUF.

Robert, A-D. (2005). Les professeurs des classes élémentaires des lycées et leur représentation: crépuscule et postérité d'une idéologie catégorielle. In Caspard P, Luc JN, Savoie P. Lycées, lycéens, lycéennes, deux siècles d'histoire. (pp.317-329). Lyon: INRP.

Robert, A-D. (2014). La pensée critique des enseignants, introduction à une notion peu commune. In Espinosa, J, Robert, A. Pensar la educación de otra manera. Epistemología social y pensamiento crítico. México: Juan Pablos Editor.

Sensévy, G. (2011). Le sens du savoir. Eléments pour une théorie de l'action conjointe en didactique. Louvain La Neuve: De Boeck.

Thierry, A. (1923). Réflexion sur l'éducation. Paris: Librairie du travail.

Testanière J. \& Pinthiaux P. (1979). Le conseil des maîtres, le rôle des instituteurs en 1969 dans l'orientation des élèves. Revue Française de Sociologie, XX, 703-732.

Thomas, J.P. (1998). De la fâcheuse influence d'esprits mal équilibrés. Communication 66, 147-164.

Vial, J. (1980). Compte rendu de lecture de Gloton, R. Au pays des enfants masqués. Revue française de pédagogie, 52, 69-71.

Noëlle Monin

Maître de conférences émérite à l'université Lyon 1 ESPE. Elle contribue aux recherches en histoire de l'éducation de l'EA4571 Education, Culture

Politiques, Lyon2.

Email: noelle.monin@laposte.net

Adresse:

Noëlle Monin

208, rue du 11 novembre 1918

38500 Coublevie

Data de submissão: Março 2018

Data de avaliação: Agosto 2018

Data de publicação: Abril 2019 